\title{
Gut microbial beta-glucuronidase and glycerol/diol dehydratase activity contribute to dietary heterocyclic amine biotransformation
}

Jianbo Zhang ${ }^{1,3}$, Christophe Lacroix¹, Esther Wortmann ${ }^{1}$, Hans-Joachim Ruscheweyh², Shinichi Sunagawa², Shana J. Sturla ${ }^{1}$ and Clarissa Schwab ${ }^{1 *}$ (i)

\begin{abstract}
Background: Consuming red and processed meat has been associated with an increased risk of colorectal cancer (CRC), which is partly attributed to exposure to carcinogens such as heterocyclic amines (HCA) formed during cooking and preservation processes. The interaction of gut microbes and HCA can result in altered bioactivities and it has been shown previously that human gut microbiota can transform mutagenic HCA to a glycerol conjugate with reduced mutagenic potential. However, the major form of HCA in the colon are glucuronides (HCA-G) and it is not known whether these metabolites, via stepwise microbial hydrolysis and acrolein conjugation, are viable precursors for glycerol conjugated metabolites. We hypothesized that such a process could be concurrently catalyzed by bacterial beta-glucuronidase (B-GUS) and glycerol/diol dehydratase (GDH) activity. We therefore investigated how the HCA-G PhIP-N2- $\beta$-D-glucuronide (PhIP-G), a representative liver metabolite of PhIP (2-Amino-1-methyl-6phenylimidazo [4,5-b] pyridine), which is the most abundant carcinogenic HCA in well-cooked meat, is transformed by enzymatic activity of human gut microbial representatives of the phyla Firmicutes, Bacteroidetes, and Proteobacteria.
\end{abstract}

Results: We employed a combination of growth and enzymatic assays, and a bioanalysis approach combined with metagenomics. B-GUS of Faecalibacterium prausnitzii converted PhIP-G to PhIP and GDH of Flavonifractor plautii, Blautia obeum, Eubacterium hallii, and Lactobacillus reuteri converted PhIP to PhIP-M1 in the presence of glycerol. In addition, B-GUS- and GDH-positive bacteria cooperatively converted PhIP-G to PhIP-M1. A screen of genes encoding B-GUS and GDH was performed for fecal microbiome data from healthy individuals $(n=103)$ and from CRC patients $(n=53)$, which revealed a decrease in abundance of taxa with confirmed GDH and HCA transformation activity in CRC patients.

Conclusions: This study for the first time demonstrates that gut microbes mediate the stepwise transformation of PhIP$\mathrm{G}$ to PhIP-M1 via the intermediate production of PhIP. Findings from this study suggest that targeted manipulation with gut microbes bearing specific functions, or dietary glycerol supplementation might modify gut microbial activity to reduce HCA-induced CRC risk.

Keywords: Heterocyclic amines (HCA), Glycerol/diol dehydratase, Reuterin, $\beta$-Glucuronidase, Faecalibacterium prausnitzii, Eubacterium hallii

\footnotetext{
* Correspondence: clarissa.schwab@hest.ethz.ch

'Department of Health Sciences and Technology, ETH Zürich, Zürich,

Switzerland

Full list of author information is available at the end of the article
}

(c) The Author(s). 2019 Open Access This article is distributed under the terms of the Creative Commons Attribution 4.0 International License (http://creativecommons.org/licenses/by/4.0/), which permits unrestricted use, distribution, and reproduction in any medium, provided you give appropriate credit to the original author(s) and the source, provide a link to the Creative Commons license, and indicate if changes were made. The Creative Commons Public Domain Dedication waiver (http://creativecommons.org/publicdomain/zero/1.0/) applies to the data made available in this article, unless otherwise stated. 


\section{Background}

Regular consumption of cooked and processed meat increases the risk for colorectal cancer (CRC) due to the prolonged exposure to meat-derived carcinogens such as 2-amino-1-methyl-6-phenylimidazo [4,5-b] pyridine (PhIP), 2-amino-3,8-dimethylimidazo [4,5-f]quinoxaline (MeIQx) and other heterocyclic amines (HCA) [1, 2]. After being absorbed in the gut, HCA are activated by liver cytochrome P450 enzymes and $N$-acetyltransferase or sulfotransferase to form acetyl esters or sulfates $[3,4]$. These conjugates are not stable and decompose to nitrenium intermediates, which can form DNA adducts [5], induce DNA mutation in bacterial and mammalian cell-based genotoxicity assays [6], and induce tumours in the large intestine of rats [7]. Alternatively, $N$-glucuronidation at the $N^{2}$ - or 3 -positions of $\mathrm{HCA}$ or $\mathrm{OH}-\mathrm{N}-\mathrm{HCA}$, mainly catalysed by uridine diphosphate (UDP)-glucuronosyltransferases in the liver, competes with the activation pathway and results in the formation of inactive glucuronide conjugates including HCA- $N^{2}-\beta$-D-glucuronide (HCA-G), HCA-3- $\beta$-D-glucuronide, $\mathrm{OH}-N-\mathrm{HCA}-\mathrm{G}$, and $\mathrm{OH}-N-\mathrm{HCA}-3-\beta-\mathrm{D}$-glucuronide [8-11]. Of these metabolites, $N^{2}$-glucuronide conjugates of
$\mathrm{HCA}$ and $\mathrm{OH}-\mathrm{N}-\mathrm{HCA}$ are the major metabolites in human hepatocytes, accounting for up to $71 \%$ of the HCA dose [12]. These glucuronide conjugates, together with the unchanged HCA, enter the urine or colon with the bile (major route) (Fig. 1) [13].

In the intestine, glucuronide conjugates can be hydrolyzed by bacterial beta-glucuronidases (B-GUS, EC 3.2.1.31), which liberate potentially bioactive aglycones [14]; the release of $\mathrm{OH}-\mathrm{N}$-products could potentially lead to mutagenicity via interaction with colon epithelial cells. Additionally, for HCA-G, HCA may be taken up into the liver, where it may be activated and potentially damage molecular targets such as DNA, or it may be converted back to HCA-G, re-entering the intestine (Fig. 1), while contributing to enterohepatic circulation which can prolong the duration of HCA exposure. Indeed, there was a second peak of PhIP and its liver metabolites in fecal and urine samples of human subjects $48-72 \mathrm{~h}$ after ingesting well-cooked chicken containing 0.9-5 $\mu$ g PhIP [15].

While the capacity of human gut microbiota to hydrolyze HCA-G is poorly understood, there are

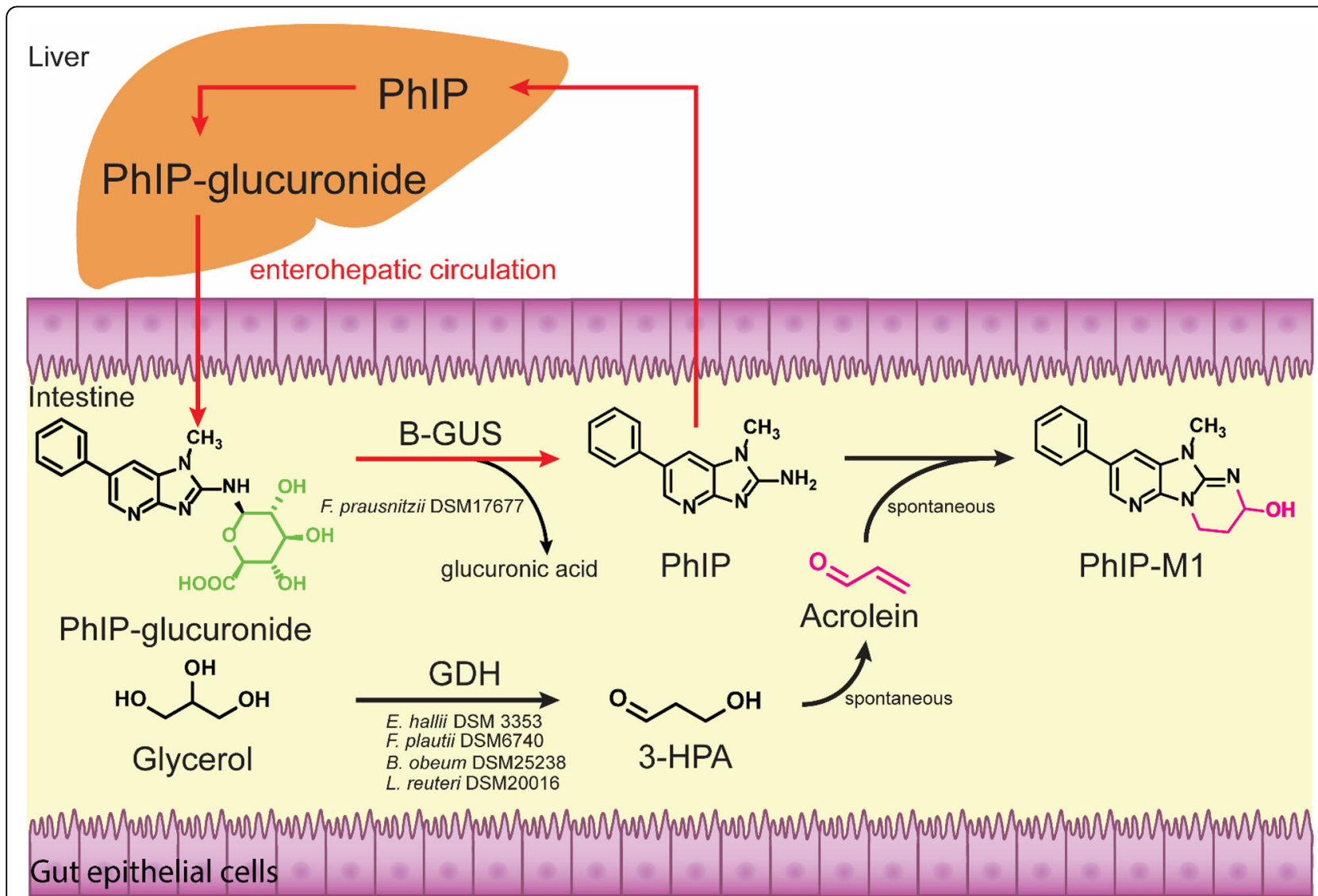

Fig. 1 Bacterial activities contributing to activation and detoxification processes governing the enterohepatic disposition of PhIP. B-GUS, $\beta$ glucuronidase; GDH, glycerol/diol dehydratase; 3-HPA, 3-hydroxypropionaldehyde 
several studies supporting that human gut microbiota can transform HCA to glycerol conjugates. For example, formation of the PhIP microbial metabolite 7hydroxy-5-methyl-3-phenyl-6,7,8,9-tetrahydropyrido [3 ,2':4,5]imidazo [1,2-a]pyrimidin-5-ium chloride (PhIP-M1) has been observed during growth of the gut microbes Lactobacillus reuteri, Eubacterium hallii, and strains of Enterococcus in the presence of glycerol (Fig. 1) [16-19]. The formation of HCA-M1 from PhIP involves a multi-step process: (1) the enzymatic reduction of glycerol to 3-hydroxy-propionaldehyde (3-HPA) by coenzyme $\mathrm{B}_{12}$-dependent glycerol/diol dehydratases (GDH, EC 4.2.1.28 and EC 4.2.1.30), (2) the accumulation of 3-HPA, (3) spontaneous dehydration of 3-HPA to form acrolein, and (4) the chemical reaction of acrolein with HCA [18]. Consistent with the glycerol conjugation reaction blocking the primary amino group of HCA, PhIP-M1 and 9-hydroxyl-2,7-dimethyl-7,9,10,11tetrahydropyrimido[20,10:2,3] imidazo [4,5-f]quinoxaline (MeIQx-M1) have lower mutagenicity than PhIP and MeIQx in the Ames test with S9 activation [15, 20] and were not observed to induce cell malignant transformation of BALB/c 3 T3 cells [20, 21]. Some strains further metabolize 3-HPA to 1,3-propanediol (1,3-PD) [22]. A second substrate of GDH is 1,2-propanediol (1,2-PD), a fermentation product of fucose and rhamnose [22, 23]. GDH reduces 1,2-PD to propionaldehyde, which can be further metabolized to propionate and 1-propanol as end products [22, 24].

Given that B-GUS is a regular enzymatic activity of gut microbiota [14], and that gut microbes that possess GDH can convert HCA to HCA-M1, we hypothesized that HCA-G hydrolysis and glycerol-dependent HCA conjugation catalyzed by bacterial GDH and B-GUS concurrently occur (Fig. 1). We tested this hypothesis by performing co-culture fermentations with B-GUS and GDH positive gut microbes using PhIP- $N^{2}-\beta$-D-glucuronide (PhIP-G) as a representative HCA-glucuronide. PhIP-G is the second most abundant $N$-linked PhIP-glucuronide in humans [12]. Compared to OH- $N^{2}$-PhIP glucuronide conjugates, the lack of hydroxyl moiety at the exocyclic amino group yields a primary amine after de-conjugation. This primary amine is key for glycerol conjugation [18], therefore, PhIP-G is an appropriate model metabolite for the stepwise reaction by B-GUS and GDH positive strains. As HCA intake has been linked to CRC, we investigated the potential of fecal microbiomes to release PhIP from PhIP-G and convert it to HCA-M1. Metagenomes $(n=156)$ of healthy and CRC patients [25] were screened for gene abundance and contributing taxa of $b$-gus and $g d h$. The results provide a mechanistic model of how gut microbiota might influence PhIP disposition and modulate carcinogenesis risk.

\section{Results}

\section{Strain selection}

We aimed to investigate GDH and B-GUS activity of gut microbes in single and co-cultures. Representative strains of species predicted to possess GDH activity were chosen based on a previous study, which used fecal metagenomes to identify species harboring gdh [24], Table 1). Gut microbes harboring $b$-gus were identified by Dabek et al. [26]. All strains were tested for both GDH and B-GUS activity.

\section{Strains with active GDH}

To determine strains with active GDH, growth capacity and metabolic activity of the selected strains (Table 1) were assessed. Incubations were performed in anaerobically prepared yeast-casitone-fatty acid (YCFA) [28] medium at $37^{\circ} \mathrm{C}$ in the presence of glycerol or 1,2-PD $(50 \mathrm{mM})$, or glucose $(50 \mathrm{mM})$ as a control. We previously showed that the presence of active GDH leads to both formation of propionate and 3-HPA from 1,2-PD and glycerol, respectively, during growth [24]. Thus, substrate utilization and major metabolite production (i.e. formate, acetate, propionate, lactate, butyrate, and 1,3-PD) were determined using high pressure liquid chromatography with a refractive index detector (HPLC-RI). All strains grew in the presence of glucose and utilized the

Table 1 Strains used, and presence of glycerol/diol dehydratase $(\mathrm{GDH})$ and $\beta$-glucuronidase (B-GUS) encoding genes. The presence of $g$ dh was predicted by metagenome analysis of human feces [24] and was confirmed for the used strains based on genome analysis (https://www.ncbi.nlm.nih.gov/genome/microbes/). The presence of b-gus was predicted by Dabek et al. [26] and Mclntosh et al. [27]

\begin{tabular}{llll}
\hline Strain name & Strain ID & gdh & b-gus \\
\hline Blautia obeum & DSM 25238 & + & - \\
Eubacterium eligens & DSM 3376 & - & + \\
Eubacterium hallii & DSM 3353 & + & - \\
Faecalibacterium prausnitzii & DSM 17677 & - & + \\
Flavonifractor plautii & DSM 6740 & + & - \\
Intestinimonas butyriciproducens & DSM 26588 & + & - \\
Lactobacillus reuteri & DSM 20016 & + & - \\
Roseburia hominis & DSM 16839 & - & + \\
Roseburia intestinalis & DSM 14610 & - & + \\
Ruminococcus gnavus & ATCC 29149 & + & - \\
Veillonella dispar & ATCC 17748 & + & - \\
Bacteroides fragilis & ATCC 25285 & - & + \\
Citrobacter freundii & CB 36 & + & - \\
Klebsiella pneumoniae & CB 35 & + & - \\
\hline
\end{tabular}

+/- gene encoding the indicated enzyme is present/absent in the

representative genome. All strains are commercially available except $C$. freundii and K. pneumoniae, which were obtained in-house collection of Laboratory of Food Biotechnology, ETH Zurich 
provided substrate with the exception of Intestinimonas butyriciproducens, which nonetheless produced butyrate (Fig. 2, Additional file 1: Table S1). Moreover, six strains, i.e. L. reuteri, E. hallii, Blautia obeum, Flavonifractor plautii, Ruminococcus gnavus, and Klebsiella pneumoniae were found to also grow in the presence of 1,2-PD (Fig. 2) and to produce propionate (Table 2). E. hallii, F. plautii, and K. pneumoniae used significantly $(p<0.05)$ more $1,2-\mathrm{PD}$ and produced significantly $(p<0.05)$ higher amounts of propionate compared to the other strains (Table 2). Other fermentation metabolites formed were acetate (0.8-9.5 mM, by B. obeum, F. plautii, R. gnavus, and K. pneumoniae), formate (1.0-5.3 $\mathrm{mM}$, by $E$. hallii, $R$. gnavus, and $K$. pneumoniae), and butyrate (4.1-4.3 $\mathrm{mM}$, by $E$. hallii and $F$. plautii). Of the six strains discovered to use 1,2-PD, five strains, i.e. B. obeum, E. hallii, F. plautii, L. reuteri, and K. pneumoniae, also metabolized glycerol. Finally, four of these, i.e. $K$. pneumoniae, E. hallii, F. plautii, and L. reuteri, produced 1,3-PD (Table 2). Glycerol consumption and 1,3 -PD formation were significantly $(p<0.05)$ higher for $K$. pneumoniae. Other metabolites produced were formate (5.3-17.5 mM, by $K$. pneumoniae), acetate (5.2-10.7 mM, by F. plautii and K. pneumoniae), and butyrate (4.3-7.8 mM, by F. plautii) (Table 2). Veillonella dispar and Roseburia hominis used glycerol, but produced mainly formate and acetate, and no 1,3-PD (Fig. 2 and Table 2). Citrobacter freundii did not use the carbon substrates supplied and likely formed formate and acetate from other components of the YCFA medium (Additional file 1: Table S1). These results suggest that six strains, i.e. B. obeum, $E$. hallii, F. plautii, L. reuteri, $R$. gnavus, and $K$. pneumoniae, were able to catalyze the reduction of
glycerol/1,2-PD, presumably mediated by GDH, and were therefore examined further for their capacity to convert the mutagenic PhIP to a non-mutagenic PhIP-M1 [16].

\section{GDH-positive bacteria convert PhIP to PhIP-M1}

To investigate whether bacteria that possess GDH activity are able to mediate PhIP to PhIP-M1 conversion, $B$. obeum, F. plautii, R. gnavus, and $K$. pneumoniae were incubated with $200 \mathrm{nM}$ PhIP in YCFA medium in the presence of $50 \mathrm{mM}$ glycerol. E. hallii and L. reuteri were used as positive controls. Levels of PhIP and PhIP-M1 were monitored using nano flow liquid chromatography electrospray ionization tandem mass spectrometry (nanoLC-ESI-MS ${ }^{2}$ ). As anticipated, E. hallii and L. reuteri converted PhIP to PhIP-M1, and F. plautii and B. obeum were newly identified as having the capacity to convert PhIP to PhIP-M1 (Fig. 3). For example, a LC-MS peak corresponding to PhIP-M1 appeared after $24 \mathrm{~h}$ incubation of PhIP with F plautii (Fig. 3a, Additional file 1: Figure S1), but not for $R$. gnavus (Fig. $3 \mathrm{~b}$ ) or $K$. pneumoniae (data not shown). $F$. plautii had a similar transformation efficiency to $L$. reuteri and E. hallii (43-60\% PhIP was converted to PhIP-M1 in $24 \mathrm{~h}$ ), which was higher than that of $B$. obeum $(8 \pm 1 \%)$ (Fig. 3c). These results indicate that not all GDH positive strains release sufficient amounts of acrolein to transform PhIP.

\section{B-GUS-positive bacteria actively convert PhIP- $N^{2}-\beta$-D- glucuronide (PhIP-G) to PhIP and/or PhIP-M1}

Having established the capacity of known and new human gut microbes with active GDH to convert PhIP to PhIP-M1, we addressed whether PhIP-G could be accessible to selected gut microbes by the action of

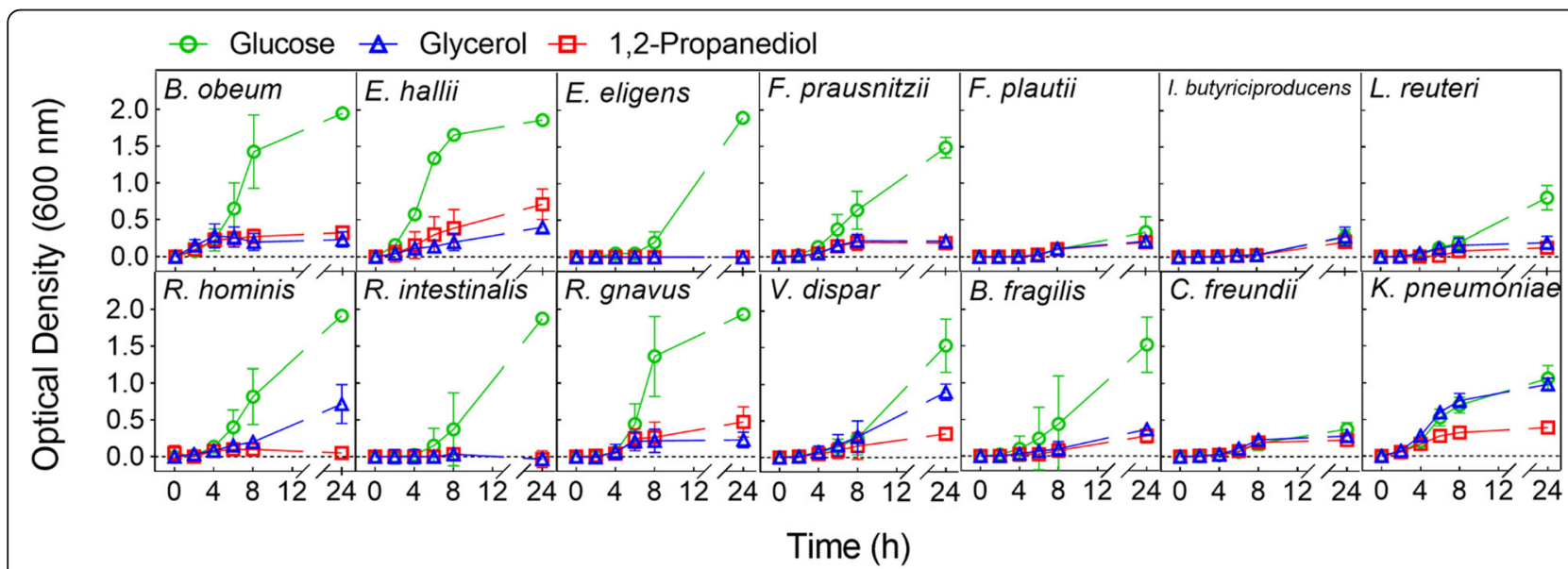

Fig. 2 Bacterial growth in the presence of glucose, glycerol, and 1,2-propanediol. Strains were grown in YCFA medium supplied with glucose (green circle), glycerol (blue triangle) or 1,2-propanediol (red rectangle) (each added at $50 \mathrm{mM}$ ) at $37^{\circ} \mathrm{C}$ for $24 \mathrm{~h}$. Optical density (OD) was measured at $600 \mathrm{~nm}$ 
Table 2 Substrate utilization and metabolite production of single strains in the presence of glycerol or 1,2-propanediol. Strains were grown in anaerobic YCFA supplied with glycerol or 1,2-propanediol (1,2-PD) (both $50 \mathrm{mM}$ ) at $37^{\circ} \mathrm{C}$ for $24 \mathrm{~h}$. E. eligens did not grow and utilize glycerol and 1,2-PD. n.d. = not determined; $n=$ number of biological replicates. Values are presented as mean \pm standard deviation

\begin{tabular}{|c|c|c|c|c|c|c|c|c|}
\hline \multirow[t]{2}{*}{ Strain $^{a}$} & \multirow[t]{2}{*}{ Substrate } & \multirow[t]{2}{*}{$n$} & \multirow{2}{*}{$\begin{array}{l}\text { Substrate } \\
\text { utilization } \\
(\mathrm{mM})\end{array}$} & \multicolumn{5}{|c|}{ Metabolite production (mM) } \\
\hline & & & & Formate & Acetate & Propionate & Butyrate & 1,3-PD \\
\hline \multirow[t]{2}{*}{ B. obeum } & $1,2-P D$ & 4 & $-17.9 \pm 4.3^{\mathrm{A}^{*}}$ & $0^{A}$ & $1.5 \pm 1.7^{\mathrm{AB}}$ & $5.2 \pm 0.4^{\mathrm{A}}$ & $-0.2 \pm 0.6^{\mathrm{A}}$ & n.d. \\
\hline & glycerol & 6 & $-2.1 \pm 1.2^{\mathrm{a}}$ & $0^{\mathrm{a}}$ & $1.5 \pm 1.4^{\mathrm{a}}$ & $-0.6 \pm 0.4^{\mathrm{a}}$ & $0^{\mathrm{a}}$ & 0 \\
\hline \multirow[t]{2}{*}{ E. hallii } & $1,2-P D$ & 6 & $-46.6 \pm 6.4^{B}$ & $1.0 \pm 0.8^{\mathrm{A}}$ & $-1.6 \pm 7.8^{\mathrm{A}}$ & $19.3 \pm 8.0^{B}$ & $4.3 \pm 0.7^{B}$ & n.d. \\
\hline & glycerol & 6 & $-13.3 \pm 3.0^{b}$ & $0.7 \pm 1.0^{\mathrm{b}}$ & $-4.6 \pm 2.5^{b}$ & $0 \pm 0.8^{\mathrm{a}}$ & $0 \pm 0.8^{\mathrm{a}}$ & $1.9 \pm 0.7^{\mathrm{a}}$ \\
\hline \multirow[t]{2}{*}{ F. prausnitzii } & $1,2-P D$ & 2 & $-0.7^{c}$ & $0^{A}$ & $-1.4^{\mathrm{A}}$ & $0^{c}$ & $1.5^{\mathrm{A}}$ & n.d. \\
\hline & glycerol & 3 & $-1.6 \pm 0.4^{c}$ & $0.9 \pm 1.6^{\mathrm{a}}$ & $1.3 \pm 0.5^{\mathrm{a}}$ & $0.3 \pm 0.2^{\mathrm{a}}$ & $0.3 \pm 0.6^{a}$ & $0^{a}$ \\
\hline \multirow[t]{2}{*}{ F. plautii } & $1,2-P D$ & 4 & $-48.1 \pm 1.2^{B}$ & $0^{A}$ & $2.2 \pm 1.4^{\mathrm{A}}$ & $11.6 \pm 3.9^{B}$ & $4.1 \pm 0.3^{B}$ & n.d. \\
\hline & glycerol & 4 & $-9.1 \pm 3.0^{c}$ & $0^{a}$ & $5.2 \pm 0.7^{c}$ & $-0.5 \pm 0.4^{\mathrm{a}}$ & $4.3 \pm 0.5^{b}$ & $1.2 \pm 0.7^{\mathrm{a}}$ \\
\hline \multirow[t]{2}{*}{ 1. butyriciproduens } & 1,2-PD & 5 & $-1.2 \pm 2.9^{c}$ & $0^{A}$ & $2.6 \pm 2.6^{A}$ & $-0.1 \pm 0.5^{c}$ & $5.4 \pm 0.6^{B}$ & n.d. \\
\hline & glycerol & 3 & $-0.8 \pm 1.4^{\mathrm{ac}}$ & $0^{a}$ & $2.5 \pm 1.7^{\mathrm{ac}}$ & $-0.1 \pm 0.1^{\mathrm{a}}$ & $5.3 \pm 0.5^{b}$ & $3.0 \pm 0.1^{b}$ \\
\hline \multirow[t]{2}{*}{ L. reuteri } & $1,2-P D$ & 3 & $-9.8 \pm 4.2^{C}$ & $0^{A}$ & $-1.0 \pm 1.1^{\mathrm{A}}$ & $0.9 \pm 0.5^{c}$ & $0^{A}$ & n.d. \\
\hline & glycerol & 3 & $-14.4 \pm 8.8^{b}$ & $0^{a}$ & $-0.4 \pm 1.1^{\mathrm{a}}$ & $0^{\mathrm{a}}$ & $0^{\mathrm{a}}$ & $5.2 \pm 0^{b}$ \\
\hline \multirow[t]{2}{*}{ R. hominis } & $1,2-P D$ & 3 & $0^{\mathrm{D}}$ & $0^{\mathrm{A}}$ & $0^{A}$ & $0^{c}$ & $0^{\mathrm{A}}$ & n.g. \\
\hline & glycerol & 5 & $-5.9 \pm 1.1^{c}$ & $0^{\mathrm{a}}$ & $-8.5 \pm 1.3^{d}$ & $-0.5 \pm 0.2^{\mathrm{a}}$ & $7.4 \pm 1.6^{b}$ & 0 \\
\hline \multirow[t]{2}{*}{ R. intestinalis } & $1,2-P D$ & 3 & $-0.4 \pm 0.4^{C}$ & $0^{A}$ & $-0.4 \pm 0.4^{\mathrm{A}}$ & $-0.3 \pm 0.1^{c}$ & $0.4 \pm 0.2^{\mathrm{A}}$ & n.d. \\
\hline & glycerol & 3 & $-1.4 \pm 0.6^{\mathrm{ac}}$ & $0^{a}$ & $1.3 \pm 1.0^{a}$ & $0.4 \pm 0.4^{a}$ & $0.4 \pm 0.2^{a}$ & $0^{\mathrm{a}}$ \\
\hline \multirow[t]{2}{*}{ R. gnavus } & $1,2-P D$ & 3 & $-18.0 \pm 5.1^{\mathrm{A}}$ & $1.1 \pm 1.9^{\mathrm{A}}$ & $2.2 \pm 1.4^{\mathrm{AB}}$ & $7.9 \pm 2.4^{\mathrm{A}}$ & $0.2 \pm 0.3^{\mathrm{A}}$ & n.d. \\
\hline & glycerol & 3 & $-1.4 \pm 1.2^{\mathrm{a}}$ & $0^{a}$ & $0.8 \pm 1.4^{\mathrm{ae}}$ & $0^{\mathrm{a}}$ & $0^{\mathrm{a}}$ & $0.2 \pm 0.3^{\mathrm{a}}$ \\
\hline \multirow[t]{2}{*}{ V. dispar } & $1,2-P D$ & 4 & $-1.7 \pm 2.4^{\mathrm{D}}$ & $1.4 \pm 1.6^{\mathrm{A}}$ & $0 \pm 2.3^{\mathrm{AB}}$ & $0.6 \pm 0.8^{C}$ & $0^{\mathrm{A}}$ & n.d. \\
\hline & glycerol & 4 & $-4.4 \pm 1.4^{\mathrm{ac}}$ & $6.6 \pm 1.2^{c}$ & $-2.4 \pm 1.9^{\text {be }}$ & $-0.5 \pm 0.5^{\mathrm{a}}$ & $-0.2 \pm 0.4^{a}$ & $0^{a}$ \\
\hline \multirow[t]{2}{*}{ B. fragilis } & 1,2-PD & 4 & $-1.0 \pm 1.4^{C}$ & $0^{A}$ & $-0.1 \pm 1.6^{\mathrm{A}}$ & $0.3 \pm 0.5^{c}$ & $-0.4 \pm 0.4^{\mathrm{A}}$ & n.d. \\
\hline & glycerol & 3 & $-1.0 \pm 0.9^{\mathrm{ac}}$ & $0^{a}$ & $0 \pm 0.5^{\mathrm{a}}$ & $0.6 \pm 0.6^{a}$ & $0^{a}$ & $0^{\mathrm{a}}$ \\
\hline \multirow[t]{2}{*}{ C. freundii } & 1,2-PD & 3 & $-1.4 \pm 1.8^{\mathrm{D}}$ & $5.4 \pm 0.6^{B}$ & $7.2 \pm 1.2^{B}$ & $1.5 \pm 0.6^{\mathrm{D}}$ & $0^{\mathrm{A}}$ & n.d. \\
\hline & glycerol & 3 & $-2.1 \pm 3.2^{a}$ & $6.0 \pm 0.5^{c}$ & $5.3 \pm 2.0^{c}$ & $-0.8 \pm 0.8^{\mathrm{a}}$ & $0^{\mathrm{a}}$ & $0^{a}$ \\
\hline \multirow[t]{2}{*}{ K. pneumoniae } & $1,2-P D$ & 3 & $-42.7 \pm 1.1^{B}$ & $5.3 \pm 0.1^{B}$ & $3.1 \pm 1.5^{\mathrm{AB}}$ & $16.7 \pm 1.0^{B}$ & $0^{\mathrm{A}}$ & n.d. \\
\hline & glycerol & 3 & $-48.7 \pm 0.8^{d}$ & $17.5 \pm 1.2^{d}$ & $10.7 \pm 0.2^{f}$ & $0^{\mathrm{a}}$ & $0^{\mathrm{a}}$ & $16.4 \pm 3.3^{c}$ \\
\hline
\end{tabular}

*Different letters represent the significant differences $(p<0.05)$ on the substrate utilization or metabolite production between different strains using multiple comparisons with 2-way ANOVA. Capital letters are for 1,2-PD and the corresponding metabolites, small letters are for glycerol and the corresponding metabolites

B-GUS, considering its presence in the gut being largely in glucuronidated form. We confirmed that Faecalibacterium prausnitzii, $R$. hominis, and Roseburia intestinalis strains previously reported to have B-GUS activity [26], were active in our hands by means of a colorimetric assay with $p$-nitrophenol- $O-\beta$-D-glucuronide (PNP-G) as a substrate. Crude cell extracts of overnight cultures of $F$. prausnitzii $\left(0.73 \pm 0.21 \mathrm{U} \mathrm{mg}^{-1}\right.$ protein) had higher B-GUS activities than those of $R$. hominis and $R$. intestinalis $\left(0.09-0.17 \mathrm{U} \mathrm{mg}^{-1}\right.$ protein) (Additional file 1: Table S2). Eubacterium eligens had very low B-GUS activity $\left(0.02 \pm 0.00 \mathrm{U} \mathrm{mg}^{-1}\right.$ protein), and all of the other strains were B-GUS negative and not further tested (Additional file 1: Table S2). We additionally performed alignments of putative proteins of E. hallii DSM 3353 against the B-GUS databases provided by McIntosh et al. [27] and Pollet et al. [29] but did not find any significant matches in the databases which would indicate the presence of putative B-GUS proteins encoded by the genome of $E$. hallii.

To identify whether strains of $F$. prausnitzii, $R$. hominis, and $R$. intestinalis hydrolyzed PhIP-G, overnight cultures were incubated in YCFA supplied with $200 \mathrm{nM}$ PhIP-G and $50 \mathrm{mM}$ glycerol and glucose at $37^{\circ} \mathrm{C}$ for $24 \mathrm{~h}$. F. prausnitzii but not $R$. hominis and $R$. intestinalis, converted $93 \pm 0.4 \%$ of PhIP-G to PhIP during growth (Fig. 4a, c and Additional file 1: Figure S2). 


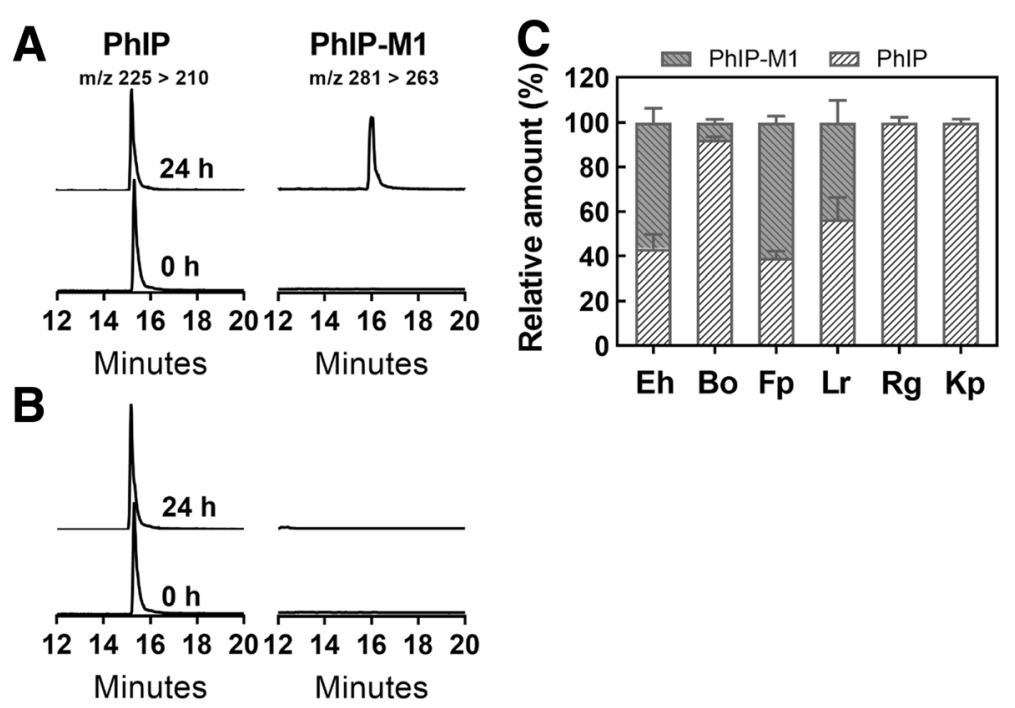

Fig. 3 Conversion of PhIP to PhIP-M1 by glycerol/1,2-propanediol utilizing bacteria during growth in YCFA. Representative ion chromatograms of PhIP and its metabolites PhIP-M1 during the growth of a F. plautii or $\mathbf{b} R$. gnavus immediately $(0 \mathrm{~h})$ or $24 \mathrm{~h}$ after inoculation. c Relative amount of PhIP and its metabolite PhIP-M1 during growth of: Eh. E. hallii $(n=3)$, Bo. B. obeum $(n=5)$, Fp. F. plautii $(n=4)$, Lr. L. reuteri $(n=3)$, Rg. R. gnavus $(n=5)$, and Kp. K. pneumoniae $(n=2)$ in YCFA with $50 \mathrm{mM}$ glycerol and PhIP $(200 \mathrm{nM})$ at $37^{\circ} \mathrm{C}$ for $24 \mathrm{~h}$. PhIP and PhIP-M1 were analysed using nanoLC-ESI-MS². Main transitions are indicated

\section{B-GUS-positive and GDH-positive bacteria cooperatively convert PhIP-G to PhIP-M1}

To test if PhIP-G can be converted to PhIP-M1 in the presence of B-GUS and GDH, we investigated co-cultures of B-GUS-positive F. prausnitzii and E. eligens, and of GDH-positive F. plautii, L. reuteri, and B. obeum. Growth in YCFA with $50 \mathrm{mM}$ glycerol, $50 \mathrm{mM}$ glucose and $200 \mathrm{nM}$ PhIP-G at $37^{\circ} \mathrm{C}$ for $24 \mathrm{~h}$ was determined using quantitative PCR (qPCR) and primers listed in Additional file 1: Table S3. Levels of PhIP-G, PhIP, and PhIP-M1 were monitored using (nanoLC-ESI-MS ${ }^{2}$ ).

Both $F$. prausnitzii and $F$. plautii grew in co-cultures, as indicated by an increase of cell counts of log 2.1 and 1.8 cells $\mathrm{ml}^{-1}$, respectively. Glucose and glycerol were utilized, and butyrate, formate, and 1,3-PD were produced (Table 3). Moreover, B-GUS activity was higher in co-cultures $\left(2.0 \pm 0.9 \mathrm{U} \mathrm{mg}^{-1}\right.$ protein) than in single cultures, and PhIP-G was indeed converted to PhIP $(52 \pm 11 \%)$ and PhIP-M1 $(27 \pm 8.5 \%)$ (Fig. 5a).

When the same experiment was performed with $F$. prausnitzii and $L$. reuteri, growth was again observed (+log 2.5 and 2.7 cells ml ${ }^{-1}$ during $24 \mathrm{~h}$ incubation, respectively), significantly $(p<0.05)$ more glucose and glycerol were used compared to $F$. prausnitzii and $F$. plautii. Butyrate, formate (Table 3), and 1,3-PD were produced, the amount of 1,3-PD was significantly higher $(p<0.05)$ compared to the $F$. prausnitzii and F. plautii co-culture. B-GUS activity was lower $\left(0.94 \pm 0.25 \mathrm{U} \mathrm{mg}^{-}\right.$ ${ }^{1}$ protein) compared to the co-culture of $F$. prausnitzii

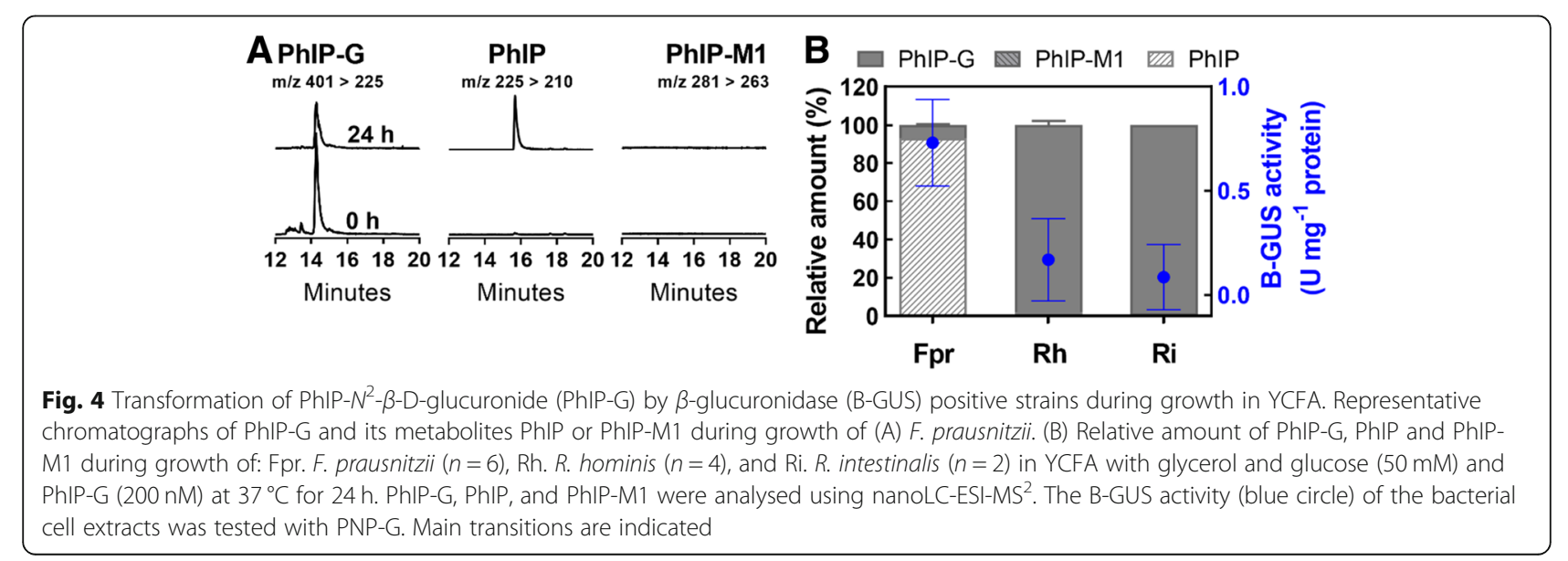


Table 3 Substrate utilization and metabolite production of co-cultures. Co-cultures were grown in YCFA supplied with glucose and glycerol (both $50 \mathrm{mM}$ ) at $37^{\circ} \mathrm{C}$ for $24 \mathrm{~h}$. Substrate utilization and metabolite production were quantified with HPLC-RI. $n=$ number of biological replicates. Values are presented as mean \pm standard deviation

\begin{tabular}{|c|c|c|c|c|c|c|c|c|}
\hline \multirow[t]{2}{*}{ Co-culture $^{a}$} & \multirow[t]{2}{*}{ Substrate } & \multirow[t]{2}{*}{$\mathrm{n}$} & \multirow{2}{*}{$\begin{array}{l}\text { Substrate } \\
\text { utilization } \\
(\mathrm{mM})\end{array}$} & \multicolumn{5}{|c|}{ Metabolite production (mM) } \\
\hline & & & & Formate & Acetate & Propionate & Butyrate & 1,3-PD \\
\hline F. prausnitzii and F. plautii & $\begin{array}{l}\text { glycerol } \\
\text { glucose }\end{array}$ & 4 & $\begin{array}{l}-11.2 \pm 6.6^{\mathrm{A}^{*}} \\
-13.4 \pm 4.4^{\mathrm{A}}\end{array}$ & $13.1 \pm 3.3^{\mathrm{A}}$ & $-6.7 \pm 3.5^{\mathrm{A}}$ & $-1.5 \pm 1.1^{\mathrm{A}}$ & $13.3 \pm 2.2^{\mathrm{A}}$ & $1.2 \pm 0.4^{\mathrm{A}}$ \\
\hline F. prausnitzii and L. reuteri & $\begin{array}{l}\text { glycerol } \\
\text { glucose }\end{array}$ & 3 & $\begin{array}{l}-26.5 \pm 0.6^{B} \\
-20.6 \pm 3.0^{B}\end{array}$ & $10.7 \pm 1.9^{\mathrm{A}}$ & $-2.0 \pm 4.9^{\mathrm{A}}$ & $-1.6 \pm 0.5^{\mathrm{A}}$ & $10.8 \pm 1.8^{\mathrm{A}}$ & $15.9 \pm 3.6^{\mathrm{B}}$ \\
\hline F. prausnitzii and B. obeum & $\begin{array}{l}\text { glycerol } \\
\text { glucose }\end{array}$ & 2 & $\begin{array}{l}-1.8 \pm 3.0^{B} \\
-23.0 \pm 1.1^{C}\end{array}$ & $11.5 \pm 1.3^{\mathrm{A}}$ & $14.4 \pm 2.8^{B}$ & $-1.6 \pm 0.4^{\mathrm{A}}$ & $9.3 \pm 2.8^{\mathrm{A}}$ & $0^{\mathrm{A}}$ \\
\hline
\end{tabular}

*Different letters represent the significant differences $(p<0.05)$ on the substrate utilization or metabolite production between different co-cultures using multiple comparisons with 2-way ANOVA

and F. plautii. PhIP-G was converted to PhIP $(51 \pm 12 \%)$ and PhIP-M1 (33 $\pm 12 \%)$ (Fig. 5b).

A third co-culture evaluated included $F$. prausnitzii and B. obeum. Only F. prausnitzii grew $(+\log 2.1$ cells

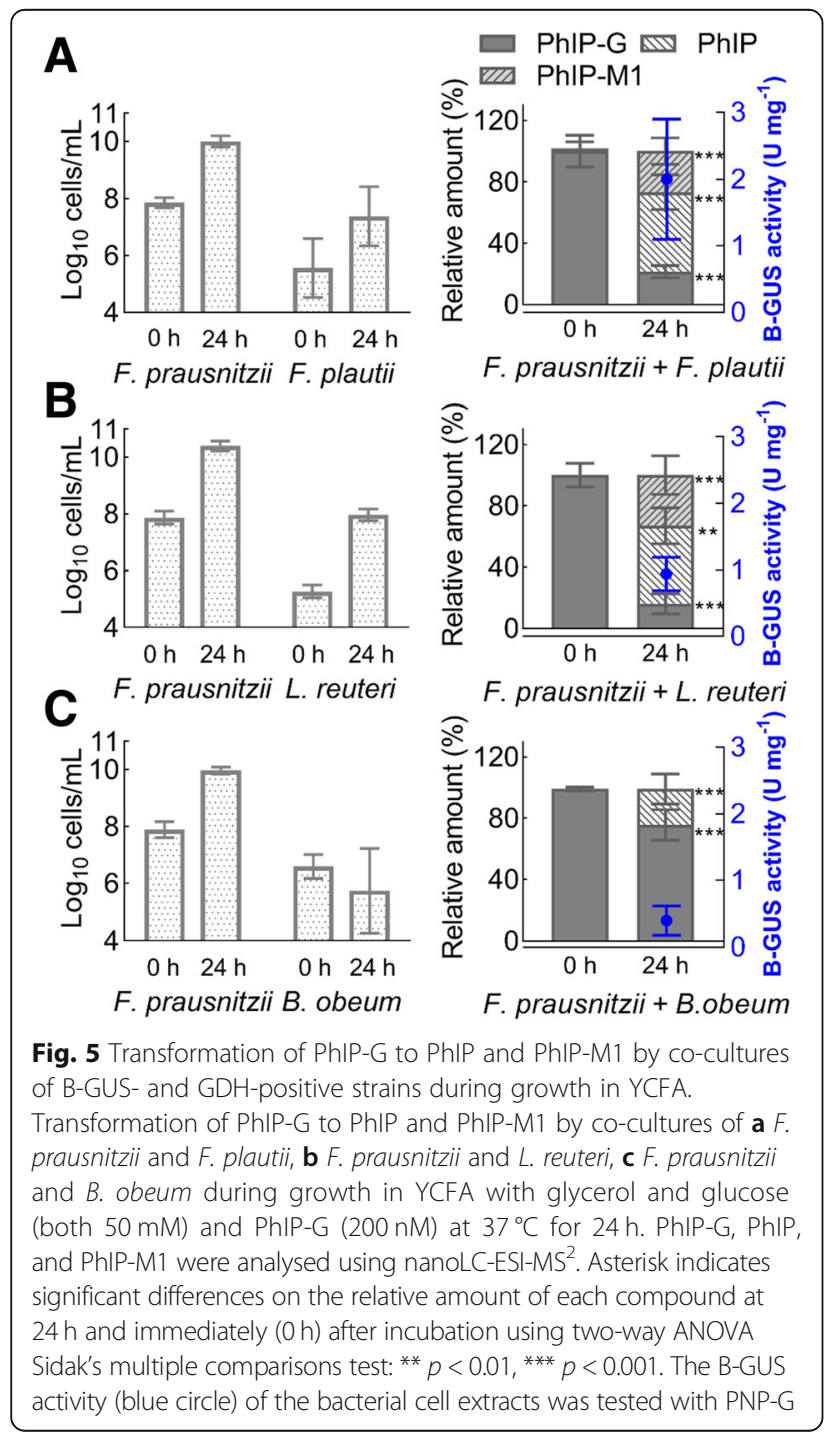

$\mathrm{ml}^{-1}$ ), utilizing glucose to produce formate, acetate, and butyrate (Table 3). There was lower B-GUS activity $\left(0.40 \pm 0.22 \mathrm{U} \mathrm{mg}^{-1}\right.$ protein) compared to the other two co-cultures, and PhIP-G was converted only to PhIP $(24.0 \pm 9.9 \%)$ (Fig. 5c). Glycerol was not used and 1,3-PD and PhIP-M1 were not detected consistent with a lack of growth of B. obeum (Fig. 5c, Table 3). In co-cultures of $E$. eligens and $B$. obeum, no PhIP-G was hydrolyzed and no PhIP and PhIP-M1 were observed (data not shown), which is in agreement with the comparatively lower B-GUS activity $\left(0.024 \pm 0.004 \mathrm{U} \mathrm{mg}^{-1}\right.$ protein $)$ than F. prausnitzii.

PhIP-M1 formation was correlated in a positive linear relationship with glycerol utilization up to $14 \mathrm{mM}$ in both single and co-cultures (Fig. 6a). At higher concentrations, the correlation plateaued. Similarly, PhIP-G hydrolysis linearly and positively correlated with B-GUS activity and plateaued at approximately $1.0 \mathrm{U} \mathrm{mg}^{-1}$ protein (Fig. 6b). Taken together, co-cultures of $F$. prausnitzii and the GDH positive $F$. plautii and $L$. reuteri were capable of catalysing the two-step process involving the release of PhIP from PhIP-G and its conjugation to form PhIP-M1.

\section{Fecal microbiome potential to hydrolyse and convert PhIP-G}

To investigate the potential of fecal microbiomes to convert PhIP-G to PhIP-M1, we screened metagenomes of healthy individuals and CRC patients (healthy, $n=103$, CRC state I-IV, $n=53$ ) of a French cohort [25] for b-gus and $g d h$ using the extensive, generic protein database RefSeq as reference. All 156 metagenomes harboured sequence homologous of $b$-gus and gdh (Fig. 7). Mean gene abundance of $b$-gus did not differ between healthy donors (mean 192.6 gene copies per thousand bacterial cells (GC), median: 181.2 GC) and CRC patients (mean 197.2 GC, median: 176.8 GC), but proportions of the main contributing phyla were significantly $(p<0.05)$ different between healthy donors (Firmicutes mean: 57.6\%, median: 59.5\%, and Bacteroidetes mean: 39.0\%, median: 


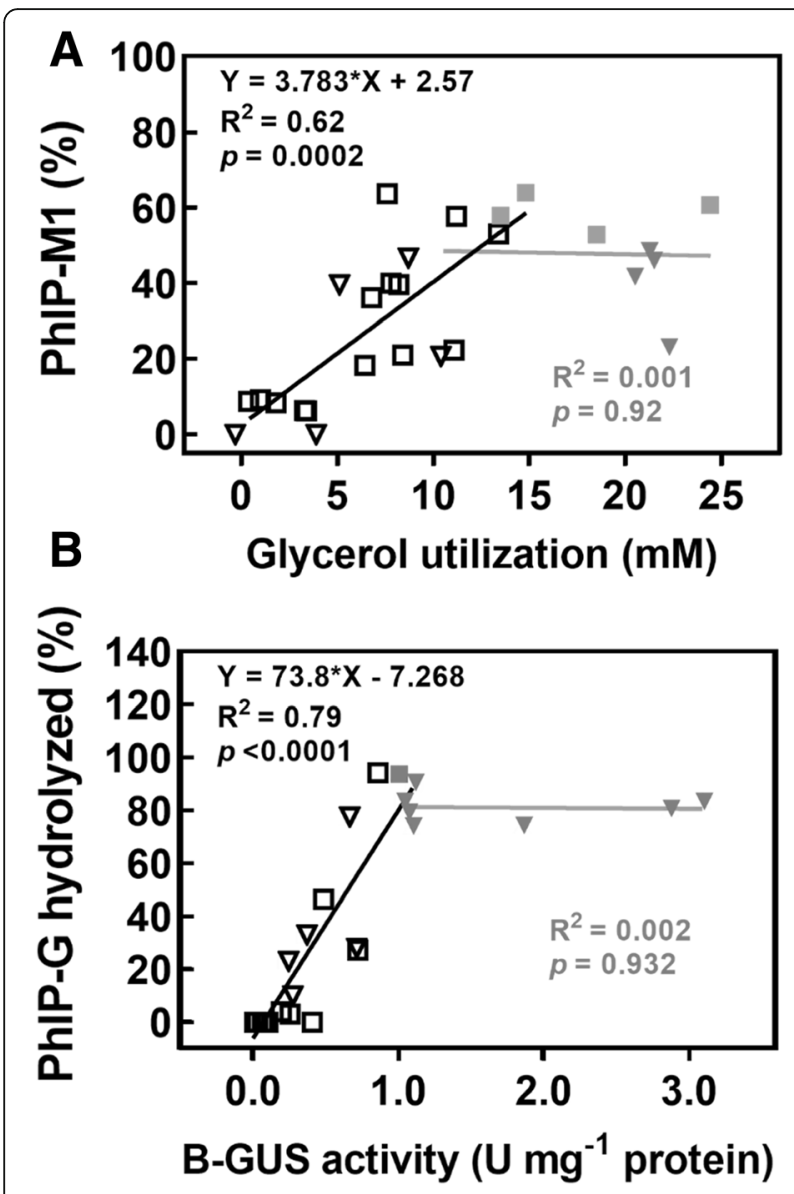

Fig. 6 Correlation of glycerol utilization and B-GUS activity with relative PhIP-M1 formation and PhIP-G hydrolysis. a correlation of glycerol utilization and PhIP-M1 formation. The proportion of PhIP-M1 was calculated as the ratio of PhIP-M1 relative to the total amount of PhIP and PhIP-M1, b correlation of B-GUS activity (determined using PNP-G) and PhIP-G hydrolysis. Data were combined from single cultures (filled and open square) and co-cultures (filled and open triangle). Grey and black symbols in (a) represent glycerol utilization above and below $14 \mathrm{mM}$, respectively. Grey and black symbols in (b) represent BGUS activity above and below $1.0 \mathrm{U} / \mathrm{mg}$ protein, respectively

39.0\%) and CRC patients (Firmicutes mean: 37.2\%, median: 35.5\%, and Bacteroidetes mean: 56.4\%, median: 59.7\%) (Fig. 7a, b).

Likewise, mean gene abundance of $g d h$ was not different between healthy donors (mean: $57.0 \mathrm{GC}$, median: $36.3 \mathrm{GC}$ ) and CRC patients (mean: 61.4 GC, median: $31.5 \mathrm{GC})$. For $g d h, E$. hallii contributed the highest proportion (mean 28.1\%), followed by B. obeum (mean 24.5\%), R. gnavus (mean: 11.0\%) and F. plautii (mean: 6.3\%) (Fig. 7c). Our activity screening covered representatives of $78 \%$ of all $g d h$ identified. The proportion of $g d h$ of taxa with confirmed HCA transformation ( $E$. hallii, F. plauttii, B. obeum and $L$. reuteri) was significantly higher in healthy donors (mean: $72.3 \%$ ) than in
CRC patients (mean: 62.3\%, Fig. 7d). This reduction of gdh of taxa with confirmed HCA transformation was observed despite converging shifts of E. hallii and F. plautii. In CRC patients, the proportion of $E$. hallii gdh (mean 28.6\%, median: $22.1 \%)$ was significantly $(p<0.05)$ lower compared to healthy donors (mean $39.6 \%$, median: $39.4 \%$ ), whereas the relative abundance of $F$. plautii $g d h$ (mean 8.6\%, median: $3.8 \%)$ was significantly $(p<0.05)$ increased (mean $3.7 \%$, median: $1.1 \%$, in healthy patients). These data indicate the potential to conjugate the glycerol metabolite acrolein with HCA may be reduced in CRC patients.

\section{Discussion}

In addition to direct physical binding, the interaction of gut microbiota and chemicals including HCA can result in a variety of products with altered bioactivities. Therefore, human gut microbiota is emerging as a decisive modulator of disease risk. In this study, we identified a strain of $F$. prausnitzii to hydrolyze PhIP-G and liberate PhIP (Fig. 1). We newly identified F. plautii and $B$. obeum as gut microbes able to convert PhIP to PhIP-M1. Cooperation of F. prausnitzii DSM 17677 with strains of $F$. plautii or $L$. reuteri were capable of converting up to one third of PhIP-G all the way to PhIP-M1. Metagenomic mining of 156 fecal microbiomes of healthy individuals and CRC patients revealed that B-GUS is approximately 10 times more abundant than $\mathrm{GDH}$, and that in CRC patients, the proportion of taxa with the capacity to transform PhIP due to GDH activity decreased.

\section{Role of B-GUS in the release of PhIP from PhIP-G}

Gut microbial enzymatic activity has been linked to intestinal diseases and drug-induced gastrointestinal disorders [30-32], and in particular, B-GUS activity has been associated with CRC development [33, 34]. Here we show that PhIP-G, a physiologically relevant and secreted inactive metabolite of the food carcinogen PhIP, can be converted to PhIP by a strain of $F$. prausnitzii (Fig. 1).

In addition to $F$. prausnitzii DSM 17677, we confirmed that $R$. hominis DSM 16839 and $R$. intestinalis DSM 14610 have, albeit lower, B-GUS activity using PNP-G as a substrate [26]. It was previously shown that expression of B-GUS is inducible for some strains, including $R$. hominis DSM 16839 and E. eligens DSM 3376 [26, 27]. Inducibility might also be a reason for the low activity observed for E. eligens as the purified protein was reported to extensively hydrolyse PNP-G [35].

Despite hydrolyzing PNP-G, neither Roseburia strain tested hydrolyzed PhIP-G, possible reasons could be the type of glucuronide linkage and structural differences in B-GUS active sites [29, 36]. PNP-G is an 


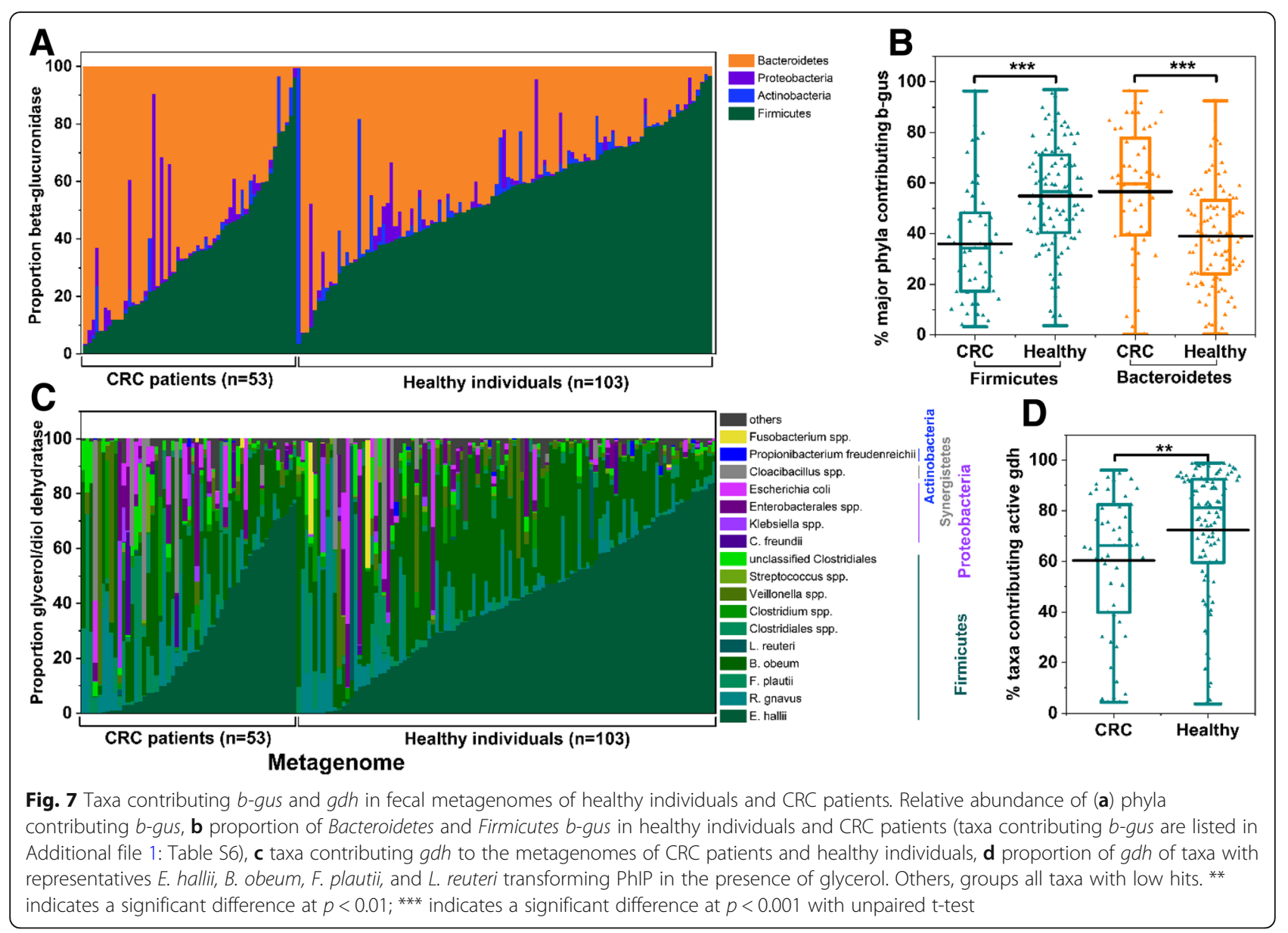

O-linked glucuronide, while the glucuronide in PhIP-G is $N$-linked, which might result in a different catalytic efficiency of B-GUS. Indeed, it has been shown that Escherichia coli B-GUS preferred O-linked glucuronides over $N$-linked glucuronides [36]. PhIP-G is the second most abundant $N$-linked PhIP glucuronide conjugate in humans after OH- $N^{2}$-PhIP-G [12]. As both PhIP glucuronide conjugates are $N$-linked, B-GUS of F. prausnitzii DSM 17677 would likely hydrolyze OH- $N^{2}$-PhIP-G. In agreement, Alexander et al. showed that strains of E. coli, K. pneumoniae, and Enterobacter aerogenes hydrolyzed OH- $N^{2}$-PhIP-G to release $\mathrm{OH}-\mathrm{N}^{2}$-PhIP, and reduced $\mathrm{OH}-\mathrm{N}^{2}-\mathrm{PhIP}$ to PhIP [9].

B-GUS activity was necessary for genotoxicity of 2-amino-3-methyl-3H-imidazo [4,5-f] quinolone (IQ), another HCA found in cooked meat, in the colon of rats monocolonized with B-GUS-deficient and wild type $E$. coli [37]. The positive correlation observed in the present study between B-GUS activity and PhIP-G hydrolysis, suggests increased PhIP exposure and residence for microbiota with higher B-GUS activity (Fig. 1).

\section{Cooperation of gut microbes to hydrolyse PhIP-G and conjugate PhIP}

Spatial confinement of relevant enzymatic activities, and substrate competition may determine the fate of PhIP-G in the microbial community. Pollet et al. predicted that many Firmicutes B-GUS proteins are likely located intracellularly [29], meaning, that in order for PhIP-G as a viable precursor to $\mathrm{PhIP}$, it needs to be transported into the bacterial cell to release PhIP. GDH is also located in the cytoplasm.

Under the conditions used here, concurrent release of PhIP from PhIP-G and its conversion to PhIP-M1 in the presence of $F$. prausnitzii and L. reuteri or F. plautii led to the formation of approximately 50\% PhIP and 30\% PhIP-M1 based on original PhIP-G levels, suggesting that bacterial interactions have the capacity to cause PhIP-G to re-enter enterohepatic circulation as well as to block the further mutagenic potential of the resulting PhIP via conversion to PhIP-M1. In a complex gut environment, proportions of PhIP and PhIP-M1 formed from PhIP-G are anticipated to vary depending on microbiota composition, the presence of strains with active B-GUS 
and GDH, and the availability of glycerol. The reason for K. pneumonia not transforming PhIP to PhIP-M1 might be because this species also metabolizes glycerol by an oxidative pathway to finally form dihydroxyacetone phosphate (DHAP), which feeds into glycolysis [38, 39].

\section{Potential of gut microbial communities to convert PhIP-G to PhIP-M1}

The $b$-gus was recently identified as a regular component of fecal metagenomes [29], indicating the potential to hydrolyze glucuronated HCA. Here we confirm that all 156 metagenomes from healthy individuals and CRC patients possessed b-gus, suggesting a global potential for HCA-G reactivation. Gene abundance of b-gus was on average 12.4 (median 5.6) and 9.7 (median 5.2) fold higher than of $g d h$ in healthy donors and CRC patients, respectively.

Compared to $b$-gus [29], there is less known concerning the presence of $g d h$ in fecal metagenomes. We observed previously that out of 10 metagenomes, all had a diverse community of strains contributing $g d h$ [24], and all of the 156 fecal metagenomes analyzed here harbored $g d h$. Both studies assigned most of the $g d h$ to $E$. hallii, $B$. obeum and R. gnavus. Consistently, fecal microbiota from 18 different individuals all possessed GDH activity indicated by $\mathrm{PhIP}$ transformation, and the activity greatly differed between individuals (1.8-96\%) [17].

Taken together, these data show that B-GUS and GDH are both constituents of the fecal microbiota, and that intestinal microbiota has the potential to both release PhIP from PhIP-G and catalyse its conversion to PhIP-M1, which effectively blocks the bioactivation of PhIP required to induce mutagenicity. The disposition of microbial metabolites of PhIP-G and PhIP may differ in healthy humans versus CRC patients on the basis of changes in the balance of metabolic potential for the hydrolysis and dehydratase processes. While mean gene abundance for each $b$-gus and gdh was similar in both groups, we observed that the proportion of contributing bacterial taxa differed in regard to $b$-gus and $g d h$. Relative abundance of Bacteroidetes b-gus increased in CRC, and Bacteroidetes were reported to mostly carry the BG-type of B-GUS [27, 40]. However, PNP-G activity and B-GUS inducibility differed in a strain-dependent manner within a panel of members of the Bacteroidetes and Firmicutes [26, 27], therefore it would be speculative to predict B-GUS activity based on the metagenome analysis presented here. Previous studies reported higher fecal B-GUS activity in CRC patients [34], however, B-GUS activity was determined using PNP-G and not HCA-G. There is no literature available concerning the hydrolysis activity of HCA-G of Bacteroidetes B-GUS suggesting that further research linking B-GUS diversity, activity and health status is stimulated by the findings presented here. Finally, the proportion of taxa that we could confirm in actively transforming PhIP to PhIP-M1 was lower in CRC than in healthy individuals. This could indeed indicate a reduced potential of the gut microbiota of CRC patients to detoxify HCA.

\section{HCA transformation versus acrolein exposure}

The formation of HCA-M1 from HCA appears to block the potential to activate it to a DNA-reactive species, consistent with data from in vitro mutagenicity studies of these compounds using activating enzymes $[17,20]$. Their relative capacities to impact cell viability has also been evaluated to address whether conversion to M1 may increase cytotoxicity. Results were mixed depending on HCA and type of cells. While HCA-M1 cytotoxicity to colon epithelium cannot be excluded, the high concentrations required to reduce cell viability are not consistent with this being a process of significant concern specifically in the context of addressing HCA mutagenicity and carcinogenesis [20, 21, 41]. Nonetheless, the $\mathrm{HCA}$ to HCA-M1 conversion process does involve the intermediate formation of acrolein [18, 20], and we recently proposed bacterial glycerol metabolism as a new endogenous source of acrolein [42]. Acrolein is an unspecific antimicrobial agent, which may influence gut microbial composition as some bacteria are more sensitive to acrolein than other taxa [18]. In addition, acrolein can cause oxidative stress and disrupt cell homeostasis in colon epithelial cells [42]. The rate of PhIP-M1 formation is proportional to acrolein concentration [18], suggesting a requirement for relatively high acrolein levels. In agreement, physiologically based pharmacokinetic modelling suggested that high levels of acrolein are required to alter the systemic exposure of the $\mathrm{HCA}$ MeIQx in human without changing the intestinal transport [43]. As acrolein itself is toxic and can be endogenous or exogenous [42, 44], further research is necessary to evaluate the toxicological relevance and overall impact on human health from microbial mediated shifts in the disposition of HCA-G, HCA, HCA-M1 and acrolein in the intestine.

\section{Conclusion}

There is a number of reports on the association of gut microbiota dysbiosis and CRC development, but little information on the role of specific bacterial metabolic activities and interaction with dietary compounds. Gut microbes processing GDH activity have the capacity to transform HCA, however, major hepatic metabolites are HCA-G, suggesting critical evaluation of HCA-G transformation is needed. Here we confirmed that gut microbes exerting B-GUS activity have the ability to hydrolyse PhIP-G to release PhIP and, those exerting GDH activity can transform free PhIP to PhIP-M1 in 
cooperation. Concurrent activity of B-GUS and GDH may lead to formation of a product with reduced mutagenic potential, however, further investigations are needed to evaluate how bacterial reactions of PhIP-G and PhIP impact health, particularly considering the intermediacy of acrolein in this process As a first step toward addressing the relevance of these activities in humans, metagenomic mining confirmed the potential of the human gut microbiome to encode B-GUS and GDH activity. These results are the first observation that the bacterial B-GUS and GDH cooperatively mediate the stepwise conversion of HCA-G to HCA-M1 via intermediate $\mathrm{HCA}$, and provide potential targets to modulate gut microbial activities for mitigating the risk of $\mathrm{HCA}$ carcinogenesis.

\section{Methods}

\section{Strains and culture conditions}

All the strains were obtained from the Deutsche Sammlung von Mikroorganismen und Zellkulturen GmbH (DSMZ, Braunschweig, Germany) or from the strain collection of the ETH Laboratory of Food Biotechnology (Table 1).

Bacteria were reactivated from a $-80^{\circ} \mathrm{C}$ stored glycerol stock and routinely cultivated in Hungate tubes using anaerobically prepared, modified YCFA medium containing $50 \mathrm{mM}$ glucose (Additional file 1: Table S4 as described previously [28].

\section{Chemicals}

PhIP and PhIP- $N^{2}-\beta$-D-glucuronide (PhIP-G) were purchased from Toronto Research Chemicals (North York, Canada). All other chemicals are listed in Additional file 1: Table S5. PhIP and PhIP-G were applied at $200 \mathrm{nM}$ which is a physiological relevant concentration considering a daily of PhIP ranging from $72 \mathrm{ng}$ [45] to $5000 \mathrm{ng}$ [15] and a colonic volume of 160 to $203 \mathrm{ml}$ in healthy adult humans [46].

\section{Bacterial cultivation}

For growth assays, $2 \%$ of an overnight culture was inoculated into $10 \mathrm{ml}$ anaerobically prepared YCFA medium containing glucose, glycerol, 1,2-PD, or glucose and glycerol (all $50 \mathrm{mM}$ ) in Hungate tubes. Optical density at $600 \mathrm{~nm}$ was monitored immediately and $2,4,6,8$, and $24 \mathrm{~h}$ after inoculation using a WPA CO 8000 Cell Density Meter (BIOLABO Scientific Instruments, Châtel-St-Denis, Switzerland). Incubation was at $37^{\circ} \mathrm{C}$ without shaking.

To test the transformation capacity of single strains, PhIP or PhIP-G (each $200 \mathrm{nM}$ ) was added and mixed thoroughly with $10 \mathrm{ml}$ YCFA medium containing glycerol or glycerol and glucose, respectively, before addition of an overnight culture (2\%). Samples (1 ml) were transferred into a $1.5-\mathrm{ml}$ tube, immediately $(\mathrm{t}=0 \mathrm{~h})$ or after $24 \mathrm{~h}$ incubation at $37^{\circ} \mathrm{C}$, centrifuged $(20,800$ $\mathrm{rcf} \times 5 \mathrm{~min}$ ), and supernatant was transferred to a new $1.5-\mathrm{ml}$ tube. Supernatants and cell pellets were stored at $-20{ }^{\circ} \mathrm{C}$ until further analysis. The experimental procedure for the co-culture study was exactly as described above, except that $1 \%(0.1 \mathrm{ml})$ of overnight culture of each bacterium was inoculated into YCFA containing glucose and glycerol. All experiments were carried out three times unless otherwise indicated.

\section{Analysis of substrate consumption and metabolite formation}

Glucose, glycerol, 1,2-PD, 1,3-PD, formate, acetate, propionate, and butyrate were quantified with HPLC-RI using external standards [28]. Supernatants were diluted 1:1 with $\mathrm{ddH}_{2} \mathrm{O}$. Analytes were separated on an Aminex HPX- $87 \mathrm{H}$ column $(300 \times 7.8 \mathrm{~mm}, 9 \mu \mathrm{m}$ particle size; Bio-Rad Laboratories AG, Cressier, Switzerland) operated at $40{ }^{\circ} \mathrm{C}$ using isocratic conditions $\left(10 \mathrm{mM} \mathrm{H}_{2} \mathrm{SO}_{4}\right.$; $\left.0.4 \mathrm{ml} \mathrm{min}^{-1}\right)$. The injection volume was $40 \mu \mathrm{l}$. Detection limits were $1 \mathrm{mM}$ for glucose, $0.9 \mathrm{mM}$ for glycerol, 0.2 $\mathrm{mM}$ for 1,2-PD and 1,3-PD, and $0.5 \mathrm{mM}$ for formate, acetate, propionate and butyrate.

\section{Analysis of B-GUS activity of crude cell extracts}

B-GUS activity of crude cell extracts was tested using para-nitrophenol- $O-\beta$-D-glucuronide (PNP-G) as a substrate. For cell extract preparation, pellets of $2 \mathrm{~mL}$ culture grown for $24 \mathrm{~h}$ in YCFA with $50 \mathrm{mM}$ glycerol were re-suspended in $100 \mu \mathrm{l}$ sodium phosphate buffer (100 $\mathrm{mM}, \mathrm{pH}$ 6.5) in a Lysing Matrix E tube (MP Biomedicals, Solon, Switzerland). Cells were disrupted using a FastPrep (MP Biomedicals) for $40 \mathrm{~s}$ at $6 \mathrm{~m} \mathrm{~s}^{-1}$ and centrifuged $(16,900 \mathrm{rcf} \times 5 \mathrm{~min})$. Supernatants were used for analysis. Cell extract (5\%) was mixed with sodium phosphate buffer containing PNP-G $(10 \mathrm{mM})$ in a 96-well plate. Absorbance $(405 \mathrm{~nm})$ was recorded immediately and after $1 \mathrm{~h}$ incubation at $37^{\circ} \mathrm{C}$ to determine the PNP released in reference to an external calibration curve. Protein concentration in the cell extract was determined with a Bradford protein assay [47].

\section{DNA isolation and $\mathrm{qPCR}$}

Genomic DNA was isolated from $0.5 \mathrm{ml}$ culture using the FastDNA SPIN Kit for Soil (MP Biomedicals). 16S rRNA gene counts were determined by qPCR using primers targeting $16 \mathrm{~S}$ rRNA of $F$. prausnitzii, B. obeum, E. hallii, or gdh of L. reuteri, and F. plautii (Additional file 1: Table S3). Reactions were conducted using a 7500 Fast Real-Time PCR System (Applied Biosystems, Zug, Switzerland) and the Kapa SYBR FAST qPCR Master Mix Kit (Labgene Scientific, Châtel-Saint-Denis, Switzerland). Thermal cycling started with a 
denaturation step at $95^{\circ} \mathrm{C}$ for $3 \mathrm{~min}$, followed by 40 cycles consisting of denaturation $\left(95^{\circ} \mathrm{C}, 3 \mathrm{~s}\right)$ and combined annealing and extension $\left(60^{\circ} \mathrm{C}, 30 \mathrm{~s}\right)$, followed by melting curve analysis. Agarose gel electrophoresis was performed to verify the specificity of amplification and amplicon size. Standard curves were prepared from 10-fold dilutions of purified PCR amplicons of the gene of interest. Linear detection range was between $\log 3$ and $\log 8$ gene copies for 16S rRNA gene of $F$. prausnitzii, between $\log 3$ and $\log 9$ gene copies for $16 \mathrm{~S}$ rRNA gene $B$. obeum, and between $\log 3$ and $\log 10$ for gdh of L. reuteri and F. plautii. A factor of 1 and 6 was used to calculate the number of cells for F. prausnitzii [48] and B. obeum [49], respectively, to account for several copies of $16 \mathrm{~S}$ rRNA gene.

Analysis of PhIP-G, PhIP, and PhIP-M1 by nano flow liquid chromatography electrospray ionization tandem mass spectrometry (nanoLC-ESI-MS ${ }^{2}$ )

PhIP-G, PhIP, and PhIP-M1 were quantified by nanospray liquid chromatography equipped with a nanoAcquity Ultra Performance LC system (Waters Corporation, Milford, MA, US) and a TSQ Vantage triple quadruple mass spectrometer (nanoLC-ESI-MS ${ }^{2}$ ). In brief, $100 \mu \mathrm{l}$ of supernatant was mixed with the internal standard 2-amino-1-methylbenzimidazole (AMBI, $20 \mu \mathrm{l}$ of $1 \mu \mathrm{M}$ ) and dried under vacuum (miVac Duo Concentrator, Genevac, Suffolk, UK). The residue was re-dissolved with $3 \times 100 \mu \mathrm{L}$ of mixture of acetonitrile:methanol (1:1), vortexed, and centrifuged $(16,900 \mathrm{rcf} \times 5 \mathrm{~min})$. The resulting supernatants were combined in a new $1.5 \mathrm{ml}$ microcentrifuge tube and vacuum-dried. The residue was dissolved in $10 \%$ acetonitrile, filtered $(0.22 \mu \mathrm{m}$ PVDF syringe filter, BGB Analytik USA LLC), and the filtrate was transferred to a LC vial with a $250-\mu$ l glass insert.

Analytes were trapped in a trap column (Symmetry C18 Trap column, $5 \mu \mathrm{m}, \mathrm{D} \times \mathrm{L} 180 \mu \mathrm{m} \times 20 \mathrm{~mm}$, Waters) at trapping conditions $\left(100 \%\right.$ solvent $\mathrm{A}\left(\mathrm{H}_{2} \mathrm{O}\right.$ with $0.1 \%$ formic acid), $4 \mu \mathrm{lmin}^{-1}, 3 \mathrm{~min}$ ). The injection volume was $0.2 \mu \mathrm{l}$. The trap column was connected to the nano analytic column (HSS T3 column, $1.8 \mu \mathrm{m}, \mathrm{D} \times \mathrm{L} 75 \mu \mathrm{m} \times$ $250 \mathrm{~mm}$, Waters) and compounds were eluted with solvent A and solvent B (acetonitrile containing 0.1\% formic acid) using the following gradient at $0.5 \mu \mathrm{l} \mathrm{min}^{-}$ ${ }^{1}: 0-10 \%$ A (0-2 min), $10-70 \%$ A (2-20 min), $70-90 \%$ A (20-20.5 min), 90\% A (20.5-28 min), followed by re-equilibrium. Positive ion spectra were recorded using the following parameters: capillary temperature, $270^{\circ} \mathrm{C}$; spray voltage, $2.1 \mathrm{kV}$; and S-lens 76 units. Compounds were monitored using the following transitions: PhIP-G, $401 \rightarrow 225$ collision energy (CE) $30 \mathrm{eV}, 401 \rightarrow 210 \mathrm{CE} 30$ $\mathrm{eV}$; PhIP, $225 \rightarrow 210 \mathrm{CE} 29 \mathrm{eV}, 225 \rightarrow 140 \mathrm{CE} 50 \mathrm{eV}$, $225 \rightarrow 115 \mathrm{CE} 48 \mathrm{eV}$; PhIP-M1, $281 \rightarrow 263 \mathrm{CE} 28 \mathrm{eV}$, $281 \rightarrow 225 \mathrm{CE} 34 \mathrm{eV}, 281 \rightarrow 210 \mathrm{CE} 38 \mathrm{eV}$; and AMBI, $148 \rightarrow 133 \mathrm{CE} 33 \mathrm{eV}$. The transitions were selected based on previous studies $[16,50]$. The collision energy for each transition was optimized by using a mixture of authentic standards. The analyte response is linear in the range of $1-100 \mathrm{nM}$ for PhIP and PhIP-M1, and 0.5-100 $\mathrm{nM}$ for PhIP-G (Additional file 1: Figure S3). System control, data acquisition and processing were performed using Thermo Xcalibur software. The limit of quantification was between $10 \mathrm{nM}$ for PhIP and PhIP-M1 and $1 \mathrm{nM}$ for PhIP-G, respectively, based on signal-tonoise ratio $(>10: 1)$.

\section{Gene abundance of fecal metagenomes for B-GUS and GDH activities}

To investigate the distribution of $b$-gus and $g d h$ in fecal metagenomes, we reanalyzed metagenomes of healthy donors $(n=103)$, and of CRC state I-IV patients $(n=53)$ of previously generated datasets from a French cohort [25]. The dereplicated gene catalogue generated in [25] was aligned against the bacterial RefSeq database (Release 85, downloaded at 04.01.2018) [51] using DIAMOND (v0.9.13, BlastX in sensitive mode) [52]. Alignments with a bitScore lower than $99 \%$ of the best alignment were removed. Furthermore, alignments with a query and reference coverage $<80 \%$ or $>130 \%$ were filtered, and those with less than $50 \%$ positives were removed. Genes aligning to $b$-gus and $g d h$ were extracted, leading to a reduced gene catalogue containing 420 candidate genes. The corresponding B-GUS were assigned to EC 3.2.1.31 while GDH were assigned to EC 4.2.1.28 and 4.2.1.30. Taxonomic annotation of candidates was derived using the RefSeq sequence information. For each metagenome candidate gene abundance was extracted from the gene length normalized gene abundance matrix [25]. Abundance was multiplied with 1000 and further normalized by median marker gene abundance $[25,53]$ to report gene copies per thousand cells (GC).

\section{Statistical analysis}

Significant differences on the relative amount of PhIP-G, PhIP, and PhIP-M1 at 24. h and immediately after incubation $(0 \mathrm{~h})$, as well as substrate utilization and metabolite production were determined using a two-way ANOVA Sidak's multiple comparisons test. Differences of the mean coding potential of $b$-gus and $g d h$ were determined using t-test.

\section{Additional file}

Additional file 1: Table S1. Substrate utilization and metabolite production of single strains in the presence of glucose $(50 \mathrm{mM})$ in YCFA medium containing acetate during growth for $24 \mathrm{~h}$. Table S2. $\beta$-Glucuronidase (B-GUS) activity of single strains was tested with the absorbance assay using PNP-G as a probe. Table S3. Primers used in this study. Table S4. Composition of YCFA medium. Table S5. Names, suppliers and identifiers of chemicals, solvents, and 
materials. Table S6. Taxa assigned to the phyla Bacteroidetes, Firmicutes, Actinobacteria and Proteobacteria contributing b-gus to fecal metagenomes of healthy individuals and colorectal cancer patients. Figure S1. Chromatograms of PhIP-G to PhIP and PhIP-M1 standard and in fermentation of $F$. plautii at $24 \mathrm{~h}$, which is referred to Fig. 3. Figure S2. Chromatograms of PhIP-G to PhIP and PhIP-M1 standard and in fermentation of $F$. prausnitzii at $24 \mathrm{~h}$, which is referred to Fig. 4. Figure S3. Representative calibration curves used for quantification of PhIP, PhIP-M1 and PhIP-G. The chemical analog AMBI served as the internal standard. (DOCX $265 \mathrm{~kb}$ )

\section{Acknowledgements}

We thank Alfonso Die for technical assistance.

\section{Funding}

This work was supported by ETH Zürich (grant ETH-41 16-1 to SJS) and the Chinese Scholarship Council (grant 201406320209 to JZ). The funding bodies were not involved in the study design, data collection, analysis, and interpretation, decision to publish, and preparation of the manuscript.

\section{Availability of data and materials}

All data generated or analyzed during this study are included in this published article.

\section{Authors' contributions}

JZ and CS conceived research and wrote the manuscript. JZ and EW performed experiments. JZ, CL, EW, SS, SJS and CS interpreted data. CL and SJS supervised the research. HJR performed bioinformatics analysis. All authors contributed to writing the manuscript. All authors read and approved the final manuscript

\section{Ethics approval and consent to participate}

Not applicable.

\section{Consent for publication}

Not applicable.

\section{Competing interests}

The authors declare that they have no competing interests.

\section{Publisher's Note}

Springer Nature remains neutral with regard to jurisdictional claims in published maps and institutional affiliations.

\section{Author details}

'Department of Health Sciences and Technology, ETH Zürich, Zürich, Switzerland. ${ }^{2}$ Department of Biology, ETH Zürich, Zürich, Switzerland. ${ }^{3}$ Present Address: Department of Biological Engineering, Massachusetts Institute of Technology, Cambridge, MA, USA.

\section{Received: 1 January 2019 Accepted: 7 May 2019}

\section{Published online: 16 May 2019}

\section{References}

1. Bouvard V, Loomis D, Guyton KZ, Grosse Y, El GF, Benbrahim-Tallaa L, et al. Carcinogenicity of consumption of red and processed meat. Lancet Oncol. 2015;16:1599-600. https://doi.org/10.1016/S1470-2045(15)00444-1.

2. Helmus DS, Thompson CL, Zelenskiy S, Tucker TC, Li L. Red meat-derived heterocyclic amines increase risk of colon cancer: a population-based casecontrol study. Nutr Cancer. 2013;65:1141-50. https://doi.org/10.1080/ 01635581.2013.834945.

3. Hein DW, Rustan TD, Ferguson RJ, Doll MA, Gray K. Metabolic activation of aromatic and heterocyclic $\mathrm{N}$-hydroxyarylamines by wild-type and mutant recombinant human NAT1 and NAT2 acetyltransferases. Arch Toxicol. 1994; 68:129-33.

4. Turesky RJ, Le Marchand L. Metabolism and biomarkers of heterocyclic aromatic amines in molecular epidemiology studies: lessons learned from aromatic amines. Chem Res Toxicol. 2011;24:1169-214.

5. Malfatti MA, Buonarati MH, Turteltaub KW, Shen NH, Felton JS. The role of sulfation and/or acetylation in the metabolism of the cooked-food mutagen 2-amino-1-methyl-6-phenylimidazo [4, 5-b] pyridine in Salmonella typhimurium and isolated rat hepatocytes. Chem Res Toxicol. 1994;7:139-47.

6. Dragsted LO, Frandsen H, Reistad R, Alexander J, Larsen JC. DNA-binding and disposition of 2-amino-1-methyl-6-phenylimidazo [4, 5-b] pyridine (PhIP) in the rat. Carcinogenesis. 1995;16:2785-93.

7. Hasegawa R, Sano M, Tamano S, Imaida K, Shirai T, Nagao M, et al. Dosedependence of 2-amino-1-methy1-6-phenylimidazo [4, 5-b]-pyridine (PhIP) carcinogenicity in rats. Carcinogenesis. 1993;14:2553-7.

8. Kaderlik KR, Minchin RF, Mulder GJ, llett KF, Daugaard-Jenson M, Teitel CH, et al. Metabolic activation pathway for the formation of DNA adducts of the carcinogen 2-amino-1-methyl-6-phenyUmidazo $[4,5-b]$ pyridine (PhIP) in rat extrahepatic tissues. Carcinogenesis. 1994;15:1703-9.

9. Alexander J, Wallin H, Rossland OJ, Solberg KE, Holme JA, Becher G, et al. Formation of a glutathione conjugate and a semistable transportable glucuronide conjugate of $\mathrm{N}$-oxidized species of 2-amino-1-methyl6phenylimidazo [4,5-b] pyridine (PhIP) in rat liver. Carcinogenesis. 1991; 12:2239-45

10. Styczynski PB, Blackmon RC, Groopman JD, Kensler TW. The direct glucuronidation of 2-amino-1-methyl-6-phenylimidazo [4,5-b] pyridine (PhIP) by human and rabbit liver microsomes. Chem Res Toxicol. 1993; 6:846-51

11. Malfatti MA, Felton JS. N-glucuronidation of 2-amino-1-methyl-6phenylimidazo [4,5-b] pyridine (PhIP) and N-hydroxy-PhIP by specific human UDP-glucuronosyltransferases. Carcinogenesis. 2001;22:1087-93.

12. Turesky RJ, Guengerich FP, Guillouzo A, Langouët S. Metabolism of heterocyclic aromatic amines by human hepatocytes and cytochrome P4501A2. Mutat Res Mol Mech Mutagen. 2002;506-507:187-95. https://doi. org/10.1016/50027-5107(02)00165-3.

13. Malfatti MA, Felton JS. Human UDP-glucuronosyltransferase $1 \mathrm{~A} 1$ is the primary enzyme responsible for the N-glucuronidation of N-hydroxy-PhIP in vitro. Chem Res Toxicol. 2004;17:1137-44.

14. Pellock SJ, Redinbo MR. Glucuronides in the gut: sugar-driven symbioses between microbe and host. J Biol Chem. 2017;292:8569-76. https://doi.org/ 10.1074/jbc.R1 16.767434.

15. Vanhaecke L, Knize MG, Noppe H, De BH, Verstraete W, Van de Wiele T. Intestinal bacteria metabolize the dietary carcinogen 2-amino-1-methyl-6phenylimidazo $[4,5-b]$ pyridine following consumption of a single cooked chicken meal in humans. Food Chem Toxicol. 2008;46:140-8. https://doi. org/10.1016/j.fct.2007.07.008.

16. Fekry Ml, Engels C, Zhang J, Schwab C, Lacroix C, Sturla SJ, et al. The strict anaerobic gut microbe Eubacterium hallii transforms the carcinogenic dietary heterocyclic amine 2-amino-1-methyl-6-phenylimidazo [4,5-b] pyridine (PhIP). Environ Microbiol Rep. 2016;8:201-9. https://doi.org/10.1111/ 1758-2229.12369.

17. Vanhaecke L, Vercruysse F, Boon N, Verstraete W, Cleenwerck I, De Wachter $M$, et al. Isolation and characterization of human intestinal bacteria capable of transforming the dietary carcinogen 2-amino-1-methyl-6-phenylimidazo [4,5-b] pyridine. Appl Environ Microbiol. 2008;74:1469-77. https://doi.org/10. 1128/AEM.02064-07.

18. Engels C, Schwab C, Zhang J, Stevens M, Bieri C, Ebert M-O, et al. Acrolein contributes strongly to antimicrobial and heterocyclic amine transformation activities of reuterin. Sci Rep. 2016;6:36246.

19. Vanhaecke L, Van Hoof N, Van Brabandt W, Soenen B, Heyerick A, De Kimpe $\mathrm{N}$, et al. Metabolism of the food-associated carcinogen 2-amino1-methyl-6-phenylimidazo [4,5-b] pyridine by human intestinal microbiota. J Agric Food Chem. 2006;54:3454-61.

20. Zhang J, Empl MT, Schwab C, Fekry MI, Engels C, Schneider M, et al. Gut microbial transformation of the dietary imidazoquinoxaline mutagen MelQX reduces its cytotoxic and mutagenic potency. Toxicol Sci. 2017;159:266-76 https://doi.org/10.1093/toxsci//kx132.

21. Nicken P, Willenberg I, von Keutz A, von Elsner L, Hamscher G, Vanhaecke L, et al. Intestinal absorption and cell transforming potential of PhIP-M1, a bacterial metabolite of the heterocyclic aromatic amine 2-amino-1-methyl6-phenylimidazo [4,5-b] pyridine (PhIP). Toxicol Lett. 2015;234:92-8.

22. Gänzle MG. Lactic metabolism revisited: metabolism of lactic acid bacteria in food fermentations and food spoilage. Curr Opin Food Sci. 2015:2:106-17.

23. Boronat A, Aguilar J. Metabolism of L-fucose and L-rhamnose in Escherichia coli: differences in induction of propanediol oxidoreductase. J Bacteriol. $1981 ; 147: 181-5$ 
24. Engels C, Ruscheweyh HJ, Beerenwinkel N, Lacroix C, Schwab C. The common gut microbe Eubacterium hallii also contributes to intestinal propionate formation. Front Microbiol. 2016;7:713.

25. Zeller G, Tap J, Voigt AY, Sunagawa S, Kultima JR, Costea PI, et al. Potential of fecal microbiota for early-stage detection of colorectal cancer. Mol Syst Biol. 2014;10:766. https://doi.org/10.15252/msb.20145645.

26. Dabek M, McCrae SI, Stevens VJ, Duncan SH, Louis P. Distribution of $\beta$ glucosidase and $\beta$-glucuronidase activity and of $\beta$-glucuronidase gene gus in human colonic bacteria. FEMS Microbiol Ecol. 2008;66:487-95.

27. McIntosh FM, Maison N, Holtrop G, Young P, Stevens VJ, Ince J, et al. Phylogenetic distribution of genes encoding $\beta$-glucuronidase activity in human colonic bacteria and the impact of diet on faecal glycosidase activities. Environ Microbiol. 2012;14:1876-87.

28. Bircher L, Schwab C, Geirnaert A, Lacroix C. Cryopreservation of artificial gut microbiota produced with in vitro fermentation technology. In press. Microb Biotechnol. 2018;11:163-75.

29. Pollet RM, D'Agostino EH, Walton WG, Xu Y, Little MS, Biernat KA, et al. An atlas of $\beta$-glucuronidases in the human intestinal microbiome. Structure. 2017;25:1-11. https://doi.org/10.1016/j.str.2017.05.003.

30. Takasuna K, Hagiwara T, Hirohashi M, Kato M, Nomura M, Nagai E, et al. Inhibition of intestinal microflora b-glucuronidase modifies the distribution of the active metabolite of the antitumor agent , irinotecan hydrochloride ( CPT-11) in rats. Cancer Chemother Pharmacol. 1998;42:280-6.

31. Louis P, Hold GL, Flint HJ. The gut microbiota, bacterial metabolites and colorectal cancer. Nat Rev Microbiol. 2014;12:661-72.

32. Weir TL, Manter DK, Sheflin AM, Barnett BA, Heuberger AL, Ryan EP. Stool microbiome and metabolome differences between colorectal cancer patients and healthy adults. PLoS One. 2013:8:e70803.

33. Takada H, Hirooka T, Hiramatsu Y, Yamamoto M. Effect of beta-glucuronidase inhibitor on azoxymethane-induced colonic carcinogenesis in rats. Cancer Res. 1982:42:331-4.

34. Kim DH, Jin $\mathrm{YH}$. Intestinal bacterial beta-glucuronidase activity of patients with colon cancer. Arch Pharm Res. 2001;24:564-7 http://www.ncbi.nlm.nih. gov/pubmed/11794536.

35. Pellock SJ, Creekmore BC, Walton WG, Mehta N, Biernat KA, Cesmat AP, et al. Gut microbial $\beta$-Glucuronidase inhibition via catalytic cycle interception. ACS Cent Sci. 2018;4:868-79.

36. Zenser TV, Lakshmi VM, Davis BB. Human and Escherichia coli $\beta$ glucuronidase hydrolysis of glucuronide conjugates of benzidine and 4aminobiphenyl, and their hydroxy metabolites. Drug Metab Dispos. 1999;27: 1064-7.

37. Humblot C, Murkovic M, Rigottier-Gois L, Bensaada M, Bouclet A, Andrieux $C$, et al. Beta-glucuronidase in human intestinal microbiota is necessary for the colonic genotoxicity of the food-borne carcinogen 2-amino-3methylimidazo [4,5-f] quinoline in rats. Carcinogenesis. 2007;28:2419-25. https://doi.org/10.1093/carcin/bgm170.

38. Barbirato F, Grivet JP, Soucaille P, Bories A. 3-Hydroxypropionaldehyde, an inhibitory metabolite of glycerol fermentation to 1,3-propanediol by enterobacterial species. Appl Environ Microbiol. 1996;62:1448-51.

39. Seo MY, Seo JW, Heo SY, Baek JO, Rairakhwada D, Oh BR, et al. Elimination of by-product formation during production of 1,3-propanediol in Klebsiella pneumoniae by inactivation of glycerol oxidative pathway. Appl Microbiol Biotechnol. 2009;84:527-34.

40. Gloux K, Berteau O, El Oumami H, Béquet F, Leclerc M, Doré J. A metagenomic $\beta$-glucuronidase uncovers a core adaptive function of the human intestinal microbiome. Proc Natl Acad Sci U S A. 2011. 108(Suppl):4539-46

41. Vanhaecke L, Derycke L, Le Curieux F, Lust S, Marzin D, Verstraete W, et al. The microbial PhIP metabolite 7-hydroxy-5-methyl-3-phenyl-6,7,8,9tetrahydropyrido $\left[3^{\prime}, 2^{\prime}: 4,5\right]$ imidazo [1,2-a]pyrimidin-5-ium chloride (PhIPM1) induces DNA damage, apoptosis and cell cycle arrest towards Caco-2 cells. Toxicol Lett. 2008;178:61-9. https://doi.org/10.1016/j.toxlet. 2008.02.004.

42. Zhang J, Sturla SJ, Lacroix C, Schwab C. Gut microbial glycerol metabolism as an endogenous Acrolein source. MBio. 2018;9:e01947-17. https://doi.org/ 10.1128/mBio.01947-17.

43. Zhang J, Empl MT, Schneider M, Schröder B, Stadnicka-Michalak J, Breves $G$, et al. Gut microbial transformation of the dietary mutagen MelQx may reduce exposure levels without altering intestinal transport. Toxicol Vitr. https://doi.org/10.1016/j.tiv.2019.04.004.
44. Moghe A, Ghare S, Lamoreau B, Mohammad M, Barve S, McClain C, et al. Molecular mechanisms of acrolein toxicity: relevance to human disease. Toxicol Sci. 2015;143:242-55. https://doi.org/10.1093/toxsci/kfu233.

45. Augustsson K, Skog K, Jägerstad M, Steineck G. Assessment of the human exposure to heterocyclic amines. Carcinogenesis. 1997;18:1931-5.

46. Pritchard SE, Marciani L, Garsed KC, Hoad CL, Thongborisute W, Roberts E, et al. Fasting and postprandial volumes of the undisturbed colon: Normal values and changes in diarrhea-predominant irritable bowel syndrome measured using serial MRI. Neurogastroenterol Motil. 2014;26:124-30.

47. Bradford MM. A rapid and sensitive method for the quantitation of microgram quantities of protein utilizing the principle of protein-dye binding. Anal Biochem. 1976;72:248-54

48. Lopez-Siles M, Martinez-Medina M, Abellà C, Busquets D, Sabat-Mir M, Duncan $\mathrm{SH}$, et al. Mucosa-associated Faecalibacterium prausnitzii phylotype richness is reduced in patients with inflammatory bowel disease. Appl Environ Microbiol. 2015;81:7582-92.

49. Yoon SH, Ha SM, Kwon S, Lim J, Kim Y, Seo H, et al. Introducing EzBioCloud: a taxonomically united database of $16 \mathrm{~S}$ rRNA gene sequences and wholegenome assemblies. Int J Syst Evol Microbiol. 2017;67:1613-7.

50. Gu D, Raymundo MM, Kadlubar FF, Turesky RJ. Ultraperformance liquid chromatography-tandem mass spectrometry method for biomonitoring cooked meat carcinogens and their metabolites in human urine. Anal Chem. 2011;83:1093-101.

51. O'Leary NA, Wright MW, Brister JR, Ciufo S, Haddad D, McVeigh R, et al. Reference sequence (RefSeq) database at NCBI: current status, taxonomic expansion, and functional annotation. Nucleic Acids Res. 2016;44:D733-45.

52. Buchfink $B$, Xie C, Huson DH. Fast and sensitive protein alignment using DIAMOND. Nat Methods. 2015;12:59-60. https://doi.org/10.1038/nmeth. 3176

53. Sunagawa S, Mende DR, Zeller G, Izquierdo-Carrasco F, Berger SA, Kultima $J R$, et al. Metagenomic species profiling using universal phylogenetic marker genes. Nat Methods. 2013;10:1196-9.

Ready to submit your research? Choose BMC and benefit from:

- fast, convenient online submission

- thorough peer review by experienced researchers in your field

- rapid publication on acceptance

- support for research data, including large and complex data types

- gold Open Access which fosters wider collaboration and increased citations

- maximum visibility for your research: over $100 \mathrm{M}$ website views per year

At $\mathrm{BMC}$, research is always in progress.

Learn more biomedcentral.com/submissions 